\title{
Post-translational regulation of sucrose transporters by direct protein-protein interactions
}

\author{
Undine Krügel ${ }^{1}$ and Christina Kühn ${ }^{2 *}$ \\ 1 Institute of Plant Biology, University of Zürich, Zürich, Switzerland \\ ${ }^{2}$ Department of Plant Physiology, Institute of Biology, University of Berlin, Berlin, Germany
}

\section{Edited by:}

John William Patrick, The University of Newcastle, Australia

\section{Reviewed by:}

Sakiko Okumoto, Virginia Tech, USA Guillaume Pilot, Virginia Tech, USA

\section{*Correspondence:}

Christina Kühn, Department of Plant Physiology, Institute of Biology, University of Berlin, Philippstrasse 13, Building 12, 10115 Berlin, Germany e-mail: christina.kuehn@biologie. hu-berlin.de
Sucrose transporters are essential membrane proteins for the allocation of carbon resources in higher plants and protein-protein interactions play a crucial role in the post-translational regulation of sucrose transporters affecting affinity, transport capacity, oligomerization, localization, and trafficking. Systematic screening for protein interactors using sucrose transporters as bait proteins helped identifying several proteins binding to sucrose transporters from apple, Arabidopsis, potato, or tomato using the split ubiquitin system. This mini-review summarizes known sucrose transporter-interacting proteins and their potential function in plants. Not all of the identified interaction partners are postulated to be located at the plasma membrane, but some are predicted to be endoplasmic reticulum-residing proteins such as a protein disulfide isomerase and members of the cytochrome b5 family. Many of the SUT1-interacting proteins are secretory proteins or involved in metabolism. Identification of actin and actin-related proteins as SUT1-interacting proteins confirmed the observation that movement of SUT1-containing intracellular vesicles can be blocked by inhibition of actin polymerization using specific inhibitors. Manipulation of expression of these interacting proteins represents one possible way to modify resource allocation by post-translational regulation of sucrose transporters.

Keywords: protein-protein interaction, sucrose allocation, membrane microdomains, detergent-resistant membrane fraction, subcellular trafficking

\section{INTRODUCTION}

Elucidation of the interactome of membrane proteins seems to be a powerful tool to gain insights into signal transduction and regulation of nutrient transport in higher plants. For Arabidopsis, a robotic screening method was established based on the split ubiquitin system in yeast (Stagljar et al., 1998) in order to systematically analyze the membrane-based interactome with potential application in fungi, plants, and metazoan (Lalonde et al., 2010). This review will rather focus on single protein-protein interactions (PPIs) of plant sucrose transporters and summarize recent research in this field published during the past 5 years.

\section{CO-LOCALIZED SUCROSE TRANSPORTERS IN THE PHLOEM CAN INTERACT WITH EACH OTHER}

Sucrose transporters from potato and tomato belonging to different phylogenetic clades (Kühn and Grof, 2010) co-localize in phloem sieve elements (SEs; Barker et al., 2000; Weise et al., 2000; Reinders etal., 2002a). Using the split ubiquitin system it was shown that SISUT1, SISUT2, and SISUT4 from tomato (Solanum lycopersicum) are able to form homo- and heterooligomers (Reinders et al., 2002a) and, even when expressed separately from different plasmids, the two halves of SISUT1 were

Abbreviations: BIFC, bimolecular fluorescence complementation; CC, companion cell; DRM, detergent-resistant membranes; MS, mass spectrometry; PDI, protein disulfide isomerase; PM, plasma membrane; PPI, protein-protein interaction; SE, sieve element; SUC, sucrose carrier; SUS, split ubiquitin system; SUT, sucrose transporter. able to reconstitute a functional transporter (Reinders et al., 2002b).

Controversial localization of Arabidopsis sucrose transporters has been reported. Whereas AtSUC2 is assumed to be localized in phloem companion cells (CCs; Stadler and Sauer, 1996), AtSUC3/SUT2 was finally localized in phloem SEs (Meyer et al., 2004), and AtSUT4 was also detected in mesophyll protoplasts (Endler et al., 2006). Nevertheless, the promoter activity of the genes encoding all three sucrose transporters was mainly detected in phloem CCs (Schulze et al., 2003). This is not unexpected, since SE-specific sucrose transporter (SUT) expression is efficiently inhibited by help of a companion-cell-specific antisense construct (Kühn et al., 1996). Arabidopsis SUTs interact with each other in yeast cells (Schulze et al., 2003). Detection of strong promoter activity of AtSUT4 in phloem minor veins (Weise et al., 2000; Schulze et al., 2003) and the detection of AtSUT4 transcripts in mesophyll cells (Endler et al., 2006) argue for the presence of AtSUT4 in both tissues.

It should be also noted that the subcellular localization of SUT/SUCs does not completely overlap with each other. It is obvious that the subcellular localization of sucrose transporters is crucial for their functionality. Whereas StSUT1 localization is primarily at the plasma membrane (PM), SISUT2 is also detectable in intracellular structures (Figure 1). SISUT2 and StSUT1 interact completely different populations of proteins (Krügel and Kühn, unpublished results) suggesting different functions. The fact that SISUT2-YFP fusion mainly localizes to intracellular membranes in 


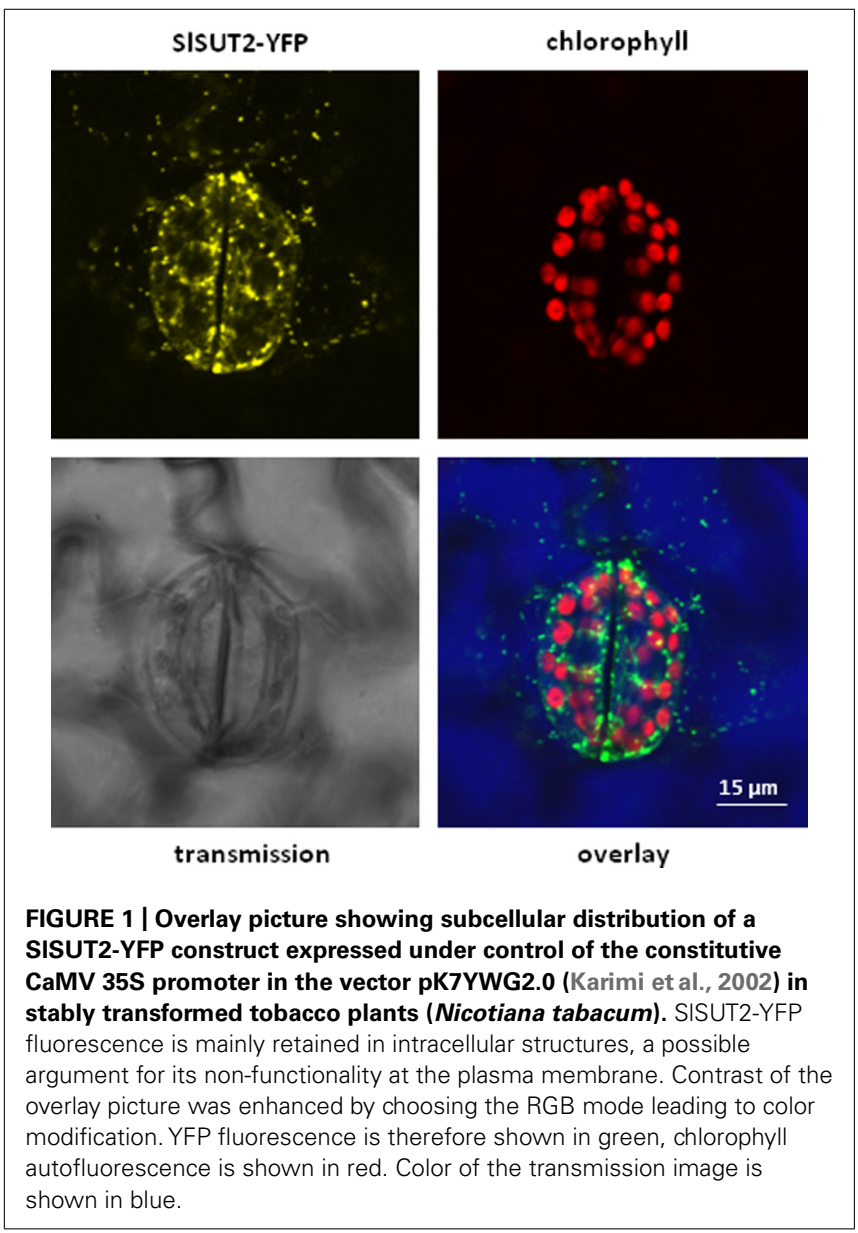

stably transformed tobacco plants (Figure 1) might be one reason for its non-functionality as sucrose transporter in heterologous expression systems. It should be taken into consideration also for members of the SUT4 family that their subcellular localization is not necessarily static and confined to one single compartment (Chincinska et al., 2013), but undergoes dynamic changes during development, degradation or initial targeting to two different compartments.

The ability of StSUT1 to form dimers was confirmed experimentally by biochemical methods such as blue native PAGE, chemical cross-linking, two-dimensional gel electrophoresis, and bimolecular fluorescence complementation (BiFC; Krügel et al., 2008). Heterologous expression of StSUT1 from Solanum tuberosum revealed redox-dependent formation of homodimers (Krügel et al., 2008). The capacity of StSUT1 and StSUT4 to form heterodimers in planta was confirmed by BiFC where heterodimers were mainly detected in the endoplasmic reticulum (ER; Krügel et al., 2012). Thus heterodimer formation between SUTs can be found even when their subcellular localization does not completely overlap.

\section{SUT1-INTERACTING PROTEINS}

The yeast split ubiquitin system has been used to systematically screen for StSUT1-interacting proteins and seven different candidate proteins have been identified: a PDI, an enolase, an aldehyde dehydrogenase, a proton pyrophosphatase, an aquaporin, Snakin1 (SN1), and an unknown protein (Krügel et al., 2012).

The attempts to confirm these interactions by coimmunoprecipitation (Co-IP) using specific antibodies or commercially available monoclonal antibodies raised against tagged and over-expressed SoSUT1 protein were not successful (Krügel et al., 2012), but large overlap was observed between proteins coprecipitated with StSUT1 and the detergent-resistant membrane (DRM) fraction of potato source leaf plasma membranes.

Immunoprecipitation was performed with either PMs from potato wild-type plants using an affinity-purified peptide antibody or alternatively with PMs from potato plants over-expressing a c-myc-tagged version of the sucrose transporter SoSUT1 from spinach (Leggewie et al., 2003) using monoclonal antibody against c-myc tag. Two candidates interact with StSUT1 from potato as well as with the over-expressed SoSUT1 from Spinacia oleracea: a plasma membrane $\mathrm{H}^{+}$-ATPase (CAA54045) and a temperatureinduced lipocalin (TIL; ABB02386). TILs are discussed to be involved in freezing tolerance of plants and/or in membrane stability and abiotic stress responses (Charron et al., 2005; Chi et al., 2009).

\section{PROTEINS INVOLVED IN SUBCELLULAR PROTEIN TARGETING}

StSUT1 was shown to undergo endocytosis in response to brefeldin A treatment and to be present in intracellular vesicles recycled at the PM. Motility of these vesicles is efficiently abolished by application of actin polymerization inhibitors such as latrunculin and actinomycin D (Liesche et al., 2010). This strongly suggests that movement of StSUT1-containing vesicles occurs in an actin-dependent manner involving the cellular cytoskeleton. Direct interaction of StSUT1 with actin and actin-related proteins strongly supports this hypothesis. The actin-related protein ARPC1/ARBC1A which was co-precipitated with StSUT1 (Krügel et al., 2012) is a component of the Arp2/3 complex implicated in the control of actin polymerization (Harries et al., 2005).

Also other StSUT1-interacting proteins identified by Co-IP are obviously involved in subcellular protein targeting and endosomal recycling at the PM. The integral membrane protein of the Yip1 family was shown to interact with the spinach SoSUT1 and is assumed to play a role in membrane trafficking. Yip1 family proteins are required for the biogenesis of ER-derived COPII vesicles in yeast and mammalian cells (Heidtman et al., 2005; Lorente-Rodriguez et al., 2009).

Other StSUT1-interacting proteins identified by Co-IP make part of the secretory pathway: Sec61 (NP_177993) is an ERlocalized heterotrimeric protein translocator involved in the transport of proteins into and out of the ER (Rapoport, 1992) and mediates the export of misfolded proteins to the cytoplasm for degradation in various organisms (Pilon et al., 1997; Schmitz et al., 2000). Sec34 (NP_177485) is well known to regulate vesicular trafficking from ER to Golgi (Loh and Hong, 2002) and might be responsible for correct secretion of SUT1 to the plasma membrane.

\section{SUT1-INTERACTING PROTEINS INVOLVED IN SIGNALING}

Many other StSUT1-interacting proteins that have been identified either by SUS or by Co-IP are metabolic enzymes or involved in signaling or both. 
The yeast Gal83 protein (CAB52141) is an important player under glucose limitation conferring specificity of the Snf1 complex to target proteins such as transcription factors (Yang et al., 1994). A correlation between Gal83 expression and assimilate transport in plants was described (Schwachtje et al., 2006).

A similar regulation of StSUT1 via phosphorylation as described for monosaccharide transporters in yeast can therefore be assumed (Carlson, 1998). Experimental data support the hypothesis of regulation of sucrose transporter activity via phosphorylation (Roblin et al., 1998; Nühse et al., 2004; Niittyla et al., 2007). 14-3-3 proteins are described to regulate many cellular processes by binding to phosphorylated proteins. The interaction of Arabidopsis 14-3-3 isoforms in response to changes in the plant nutrient status helped to identify new targets involved in nitrogen and sulfur metabolism (Shin et al., 2011). Among other sugar and mannitol transporters, AtSUC6 from Arabidopsis directly interacts with a $14-3-3 \chi$ protein of Arabidopsis suggesting that AtSUC6 is phosphorylated as well (Shin et al., 2011).

The detailed function of SUT phosphorylation remains to be elucidated. It is still unclear whether or not phosphorylation of the StSUT1 protein affects its subcellular localization or dimerization behavior.

\section{METABOLISM}

Adenosine diphosphate (ADP)-glucose pyrophosphorylase (AGPase) is the key enzyme in starch biosynthesis and forms an allosteric heterotetramer. The activity of AGPase is regulated, e.g., by sugars and light through post-translational redoxdependent dimerization of the small subunit (Hendriks etal., 2003; Geigenberger et al., 2005).

Furthermore it is known from transgenic plants with reduced expression of plant SUTs that starch levels in source leaves are dramatically increased, which can be visualized macroscopically (Bürkle et al., 1998; Hackel et al., 2006) or by electron microscopy (Bürkle et al., 1998; Schulz et al., 1998; Chincinska et al., 2008). The identification of StSUT1-interacting proteins by Co-IP revealed direct interaction of StSUT1 with glucose-1-phosphate adenyltransferase (CAA53741) which represents the large subunit of the AGPase tetramer (Krügel et al., 2012). Therefore adaptation of starch biosynthetic capacity is not only possible via sugar availability and the redox-dependent dimerization of the small AGPase subunit but also by direct PPI between StSUT1 and the large subunit of AGPase.

\section{REDOX-REGULATION: SNAKIN-1, PDI}

One of the interaction partners of StSUT1 repeatedly identified in several independent screens was SN1 (Krügel et al., 2012). SN1 is a small cysteine-rich cell wall protein from Solanum tuberosum with an assumed antimicrobial function. It belongs to the Snakin/GASA (gibberellic acid stimulated in Arabidopsis) protein family and its physiological function was recently elucidated by generation of transgenic potato plants (Nahirnak et al., 2012a,b). $\mathrm{SN} 1$ is a PM protein and silencing of SN1 expression in transgenic potato plants led to reduced plant height and leaf size, whereas the mean cell size was increased. SN1 silencing affects cell division, cell wall composition, and leaf primary metabolism, i.e., the level of intermediates of the tricarboxylic acid (TCA) cycle. Redox homeostasis of SN1-silenced plants seems to be disturbed because the level of reactive oxygen species (ROS) is increased in these plants, while the level of ascorbate is reduced (Nahirnak et al., 2012b).

Computational prediction suggests that conserved oxidized cysteines in Snakin/GASA proteins can create up to five disulfide bridges that may act catalytically, i.e., playing a role in redox regulation exhibiting antioxidant activity (Wigoda etal., 2006). In Arabidopsis, the GASA4 protein affects the level of $\mathrm{H}_{2} \mathrm{O}_{2}$ and $\mathrm{NO}$ upon wounding and participates in GA-dependent signaling of flowering induction (Rubinovich and Weiss, 2010).

It is assumed that OsGSR1, a Snakin/GASA protein from rice, plays an important role in the cross-talk of GA and brassinosteroids (BR) signaling pathways (Wang et al., 2009). The expression of OsGSR1 is induced by gibberellins (Gas) and repressed by BR. Thus, members of the SN1/GASA protein family cover aspects in plant development, beyond their assumed function in response to biotic or abiotic stresses (Nahirnak et al., 2012b).

The physiological role of StSUT1-SN1 interaction in potato remains to be elucidated. A similar phenotype as in SN1-inhibited potato is observed when the expression of another SUT-interacting protein, the protein disulfide isomerase, is inhibited in transgenic potato plants by RNA interference (RNAi) silencing (E. Eggert and C. Kühn, unpublished results). In view of the fact that StSUT1 forms dimers in a redox-dependent manner (Krügel et al., 2008) it is interesting to note that two out of seven StSUT1interacting proteins identified by SUS seem to be involved in redox homeostasis.

\section{INTERACTORS OF SUT4 MEMBERS: MdSUT1 AND AtSUT4 INTERACT WITH PROTEINS OF THE CYb5 FAMILY}

The MdSUT1 protein of Malus domestica, belonging to the SUT4 subfamily, was immunolocalized to the plasma membrane of apple phloem and parenchyma cells. A systematic SUS screen was performed using MdSUT1 and the sorbitol transporter MdSOT6 as bait proteins (Fan etal., 2009). Cytochrome b5 (Cyb5) was identified several times independently as interaction partners of MdSUT1 and of MdSOT6 (Table 1).

Cyb5s are small membrane-anchored proteins with a putative heme/steroid binding domain. They are involved in a number of oxidative reactions. Interaction between MdSUT1 and Cyb5 was confirmed by immunoprecipitation and BiFC. Since MdSUT1 was localized to the PM whereas Cyb5 is expected in the ER, electron microscopic immunolocalization was performed showing accurately that Cyb5 is localized in ER cisternae, which are connected to the PM (Fan et al., 2009).

$\beta$-Galactosidase (lacZ) activity tests in yeast have been used to quantify the strength of interaction in the presence or absence of glucose or sucrose in the medium. Increasing sucrose concentrations inhibited MdSUT1 interaction with Cyb5, whereas varying concentrations of glucose (which is not transported by MdSUT1) did not affect the strength of interaction (Fan et al., 2009).

Deletion constructs of the SUT-interacting Cyb5 revealed that the $\mathrm{C}$-terminal region containing the membrane spanning domain of Cyb5 is essential for the interaction with sugar transporters, 
Table 1 | Protein-protein interaction partners of plant sugar transporters identified by the yeast two hybrid split ubiquitin system.

\begin{tabular}{|c|c|c|c|c|}
\hline Bait protein & Localization & Prey proteins & Confirmation & Reference \\
\hline \multirow[t]{7}{*}{$\begin{array}{l}\text { StSUT1 (Solanum } \\
\text { tuberosum, SUT1 clade) }\end{array}$} & PM & Protein disulfide isomerase (PDI) & $\begin{array}{l}\text { GST pull-down, BiFC, DRM protein, FRET acceptor } \\
\text { bleaching, also interacts with SISUT2 and StSUT4 }\end{array}$ & Krügel etal. (2012) \\
\hline & & Snakin-1 & Several times $(>5)$ in independent screens & \\
\hline & & Inorganic pyro-phosphatase (PPi) & DRM protein & \\
\hline & & Tonoplast intrinsic protein (TIP) & DRM protein & \\
\hline & & Enolase & not confirmed & \\
\hline & & Aldehyde de-hydrogenase (ADH) & Several times in independent screens & \\
\hline & & Unknown protein & not confirmed & \\
\hline $\begin{array}{l}\text { MdSUT1 (Malus domestica, } \\
\text { SUT4 clade) }\end{array}$ & PM & Cytochrome b5 (Cyb5) & BiFC, Co-IP, identified in 17 independent colonies & Fan et al. (2009) \\
\hline $\begin{array}{l}\text { MdSOT6 (Malus domestica, } \\
\text { sorbitol transporter) }\end{array}$ & PM & Cyb5 & BiFC, Co-IP, identified in 20 independent colonies & Fan et al. (2009) \\
\hline \multirow{5}{*}{$\begin{array}{l}\text { AtSUT4 (Arabidopsis } \\
\text { thaliana, SUT4 clade) }\end{array}$} & vacuole & Cyb5-1 & BiCF, Co-IP & Li et al. (2012) \\
\hline & & Cyb5-2 & & \\
\hline & & Cyb5-3 & & \\
\hline & & Cyb5-4 & & \\
\hline & & Cyb5-6 & & \\
\hline
\end{tabular}

whereas the $\mathrm{N}$-terminus only plays a role in the strength of the interaction. Thus, it is likely that the C-terminal part of Cyb5 interacts with the N-terminal part of MdSUT1 and that interaction takes place within the membrane. Co-expression of the Cyb5 protein with MdSUT1 in yeast increased affinity of MdSUT1 toward the substrate sucrose. Besides inhibiting PPI substitution of leucine 73 with proline abolished this increase in affinity. It was suggested that the sugar transporter-Cyb5 complex, which is strengthened under sugar starvation, promotes sugar uptake when sugar availability is limited.

In Arabidopsis, the same authors showed that AtSUT4, but not homologous transporters in the SUT1 or SUT2 subfamily, is able to interact with five different members of the Cyb5 family. Confirmation of interaction was obtained from pull-down assays and via BiFC (Li et al., 2012). Both atsut4 and cyb5-2 mutant plants show decreased sensitivity toward sucrose and glucose with respect to seed germination and it is suggested that the AtSUT4/Cyb5-2 complex might be involved in sensing or signaling not only of sucrose but also of glucose (Li et al., 2012).

\section{SUMMARY AND OUTLOOK \\ PITFALLS}

The identification of StSUT1-interacting proteins by different methods revealed completely different populations of interacting proteins. This is possibly explained by the differences of the two methods that have been used: for split ubiquitin screens, often a complete cDNA library is screened. In case of the StSUT1 split ubiquitin screen, the potato cDNA library was generated from RNA isolated and pooled from various tissues and developmental stages (Krügel et al., 2012). Thus, PPIs were demonstrated which under native conditions are not possible due to expression in different tissues, cells, developmental stages, and/or cellular compartments. PPIs determined using this method always need to be confirmed in planta in order to eliminate false-positive interaction partners.

Immunoprecipitation experiments, on the other hand, have been performed with plant extracts containing PM-enriched fractions isolated at a given developmental stage from source or sink leaves (Krügel et al., 2012). These interacting proteins are at least present at the same developmental stage and in the same isolated membrane fraction. Thus, a different population of proteins is expected for this screen of PPIs. Both sets of candidate genes identified with the two different methods need further confirmation.

\section{THE POTENTIAL ROLE OF SUBCELLULAR COMPARTMENTATION}

Elucidation of the StSUT1 interactome helped to get information about the regulation and subcellular localization of this SUT. Its presence in the DRM fraction was confirmed by liquid chromatography-tandem mass spectrometry (LC-MS/MS; Krügel et al., 2012) and its association to lipid raft-like microdomains was visualized by expression of green fluorescent protein (GFP) fusion constructs in yeast (Krügel et al., 2008). SUT1 is constantly recycled at the SE PM in an actin-dependent manner (Liesche et al., 2010). Identification of actin-related proteins and components of the secretory pathway such as Sec34 and Sec61 among the StSUT1-interacting partners support these findings. A large overlap was observed between StSUT1-interacting proteins identified by Co-IP and proteins showing reduced detergent solubility (Krügel et al., 2012).

The question is now whether association of the StSUT1 to membrane microdomains is a prerequisite for endocytosis and recycling. Methyl- $\beta$-cyclodextrin $(\mathrm{M} \beta \mathrm{CD})$ causes sterol depletion 
of the plant PM and thereby inhibits raft formation (Roche et al., 2008). The significantly increased activity of the glucose transporter GLUT1 in the presence of $\mathrm{M} \beta \mathrm{CD}$ is explained by inhibition of endocytosis thereby increasing the number of transporters at the PM (Barnes et al., 2004; Caliceti et al., 2012). It is indispensable to test the impact of M $\beta C D$ on the activity of SUT1, whose endocytosis seems to be inhibited by M $\beta C D$ similarly to GLUT1 in the mammalian system (Liesche et al., 2010).

Raft association of plant membrane proteins may also impact lateral segregation. The potassium channel AtKAT1 was localized to microdomains and a low lateral mobility was observed (Reuff etal., 2010). Cholesterol depletion of the membrane decreases lateral mobility of PM proteins (Kwik et al., 2003) suggesting that association to raft-like microdomains enhance lateral diffusion.

Interestingly, the plasma membrane lining plasmodesmata share characteristics with membrane rafts, since typical raft proteins such as glycosylphosphatidylinositol (GPI)-anchored proteins, the callose binding protein, remorin and also sphingolipids and phytosterols are enriched in the PM of plasmodesmata (Mongrand et al., 2010).

Translation of StSUT mRNAs is assumed to take place in CCs and the proteins need to be targeted to the PM of neighboring SEs (Kühn and Grof, 2010). Increased lateral mobility due to raft-like properties of the PM lining plasmodesmata might represent one possible way to facilitate correct targeting of SUTs from CC into enucleate SEs.

A recent paper, however, investigated lateral mobility of minimal membrane proteins by fluorescence recovery after photobleaching (FRAP) and points to the important role of the cell wall in immobilizing PM proteins, whereas association to membrane microdomains is assumed to play only a minor role in lateral mobility (Martiniere et al., 2012).

\section{REFERENCES}

Barker, L., Kühn, C., Weise, A., Schulz, A., Gebhardt, C., Hirner, B., et al. (2000). SUT2, a putative sucrose sensor in sieve elements. Plant Cell 12, 1153-1164.

Barnes, K., Ingram, J. C., Bennett, M. D., Stewart, G. W., and Baldwin, S. A. (2004). Methyl-beta-cyclodextrin stimulates glucose uptake in Clone 9 cells: a possible role for lipid rafts. Biochem. J. 378, 343-351. doi: 10.1042/BJ20031186

Bürkle, L., Hibberd, J. M., Quick, W. P., Kühn, C., Hirner, B., and Frommer, W. B. (1998). The H+-sucrose cotransporter NtSUT1 is essential for sugar export from tobacco leaves. Plant Physiol. 118, 59-68.

Caliceti, C., Zambonin, L., Prata, C., Vieceli Dalla Sega, F., Hakim, G., Hrelia, S., et al. (2012). Effect of plasma membrane cholesterol depletion on glucose transport regulation in leukemia cells. PLOS ONE 7:e41246. doi: 10.1371/journal.pone.0041246
Carlson, M. (1998). Regulation of glucose utilization in yeast. Curr. Opin. Genet. Dev. 8, 560-564. doi: 10.1016/S0959-437X(98)80011-7

Charron, J. B., Ouellet, F., Pelletier, M., Danyluk, J., Chauve, C., and Sarhan, F. (2005). Identification, ses of plant lipocalins. Plant Physiol. 139, 2017-2028. doi: 10.1104/pp.105. 070466

Chi, W. T., Fung, R. W., Liu, H. C., Hsu, C. C., and Charng, Y. Y. (2009). Temperature-induced lipocalin is required for basal and acquired thermotolerance in Arabidopsis. Plant Cell Environ. 32, 917 927. doi: 10.1111/j.1365-3040.2009. 01972.x

Chincinska, I., Gier, K., Krügel, U., Liesche, J., He, H., Grimm, B., et al. (2013). Photoperiodic regulation of the sucrose transporter StSUT4 affects the expression of circadian-regulated genes and ethylene production. Front. Plant Sci. 4:26. doi: 10.3389/fpls.2013.00026 expression, and evolutionary analy-

\section{SIGNALING}

Raft-like membrane compartments are regarded as organizing principle allowing attribution to protein-based sub-organellar compartments within a single cell to facilitate enzymatic reactions or cellular dynamics (Holthuis and Ungermann, 2012). Moreover, lipid rafts form cholesterol-enriched redox signaling membrane platforms providing an important driving force, e.g., for the assembly of nicotinamide adenine dinucleotide phosphate (NADPH) oxidase subunits to form (or activate) a functional enzyme complex in the PM (Jin et al., 2011). Depending on the cell type, sterol depletion of the PM using $\mathrm{M} \beta \mathrm{CD}$ either delays NADPH oxidase activation leading to decreased ROS production (Vilhardt and van Deurs, 2004), decreased NADPH oxidase activity (Rao Malla et al., 2010), or promotes activation of NADPH oxidase (Han et al., 2008).

Lipid rafts are assumed to represent signaling platforms perhaps enabling membrane proteins to get in close contact to signaling proteins, kinases, phytohormone receptors, etc. However, the question remains whether the redox-dependent dimerization of SUT proteins and phosphorylation/dephosphorylation events are facilitated if the protein is concentrated in raft-like microdomains.

Manipulation of expression of SUT-interacting proteins represents a powerful tool to impact sucrose transport activity at the post-translational level in future experiments thereby strongly affecting carbon partitioning and resource allocation within crop plants.

\section{ACKNOWLEDGMENTS}

We gratefully acknowledge Stefan Hörtensteiner and Bernhard Grimm for helpful discussions and critical reading of the manuscript, as well as Enrico Martinoia for continuous and valuable support.

Chincinska, I. A., Liesche, J., Krügel, U., Michalska, J., Geigenberger, P., Grimm, B., et al. (2008). Sucrose transporter StSUT4 from potato affects flowering, tuberization, and shade avoidance response. Plant Physiol. 146, 515-528.

Endler, A., Meyer, S., Schelbert, S., Schneider, T., Weschke, W., Peters, S. W., et al. (2006). Identification of a vacuolar sucrose transporter in barley and Arabidopsis mesophyll cells by a tonoplast proteomic approach. Plant Physiol. 141, 196-207. doi: 10.1104/pp.106.079533

Fan, R. C., Peng, C. C., Xu, Y. H., Wang, X. F., Li, Y., Shang, Y., et al. (2009). Apple sucrose transporter SUT1 and sorbitol transporter SOT6 interact with cytochrome b5 to regulate their affinity for substrate sugars. Plant Physiol. 150, 1880-1901. doi: 10.1104/pp.109.141374

Geigenberger, P., Kolbe, A., and Tiessen, A. (2005). Redox regulation of carbon storage and partitioning in response to light and sugars.
J. Exp. Bot. 56, 1469-1479. doi: 10.1093/jxb/eri178

Hackel, A., Schauer, N., Carrari, F., Fernie, A. R., Grimm, B., and Kühn, C. (2006). Sucrose transporter LeSUT1 and LeSUT2 inhibition affects tomato fruit development in different ways. Plant J. 45, 180-192.

Han, W., Li, H., Villar, V. A., Pascua, A. M., Dajani, M. I., Wang, X., et al. (2008). Lipid rafts keep NADPH oxidase in the inactive state in human renal proximal tubule cells. Hypertension 51, 481487. doi: 10.1161/HYPERTENSIONAHA.107.103275

Harries, P. A., Pan, A., and Quatrano, R. S. (2005). Actinrelated protein $2 / 3$ complex component ARPC1 is required for proper cell morphogenesis and polarized cell growth in Physcomitrella patens. Plant Cell 17, 2327-2339.doi: 10.1105/tpc. 105.033266

Heidtman, M., Chen, C. Z., Collins, R. N., and Barlowe, C. (2005). Yoslp is a novel subunit of the 
Yiplp-Yiflp complex and is required for transport between the endoplasmic reticulum and the Golgi complex. Mol. Biol. Cell 16, 1673-1683. doi: 10.1091/mbc.E04-10-0873

Hendriks, J. H., Kolbe, A., Gibon, Y., Stitt, M., and Geigenberger, P. (2003). ADP-glucose pyrophosphorylase is activated by posttranslational redox-modification in response to light and to sugars in leaves of Arabidopsis and other plant species. Plant Physiol. 133, 838-849. doi: 10.1104/pp.103.024513

Holthuis, J. C., and Ungermann, C. (2012). Cellular microcompartments constitute general suborganellar functional units in cells. Biol. Chem. 394, 151-161. doi 10.1515/hsz-2012-0265

Jin, S., Zhou, F., Katirai, F., and Li, P. L. (2011). Lipid raft redox signaling: molecular mechanisms in health and disease. Antioxid. Redox Signal. 15, 1043-1083. doi: 10.1089/ars.2010.3619

Karimi, M., Inze, D., and Depicker, A. (2002). GATEWAY vectors for Agrobacterium-mediated plant transformation. Trends Plant Sci. 7 193-195.

Krügel, U., He, H. X., Gier, K., Reins, J., Chincinska, I., Grimm, B., et al. (2012). The potato sucrose transporter StSUT1 interacts with a DRM-associated protein disulfide isomerase. Mol. Plant 5, 43-62. doi: $10.1093 / \mathrm{mp} / \mathrm{ssr} 048$

Krügel, U., Veenhoff, L. M., Langbein, J., Wiederhold, E., Liesche, J., Friedrich, T., et al. (2008). Transport and sorting of the Solanum tuberosum sucrose transporter SUT1 is affected by posttranslational modification. Plant Cell 20, 2497-2513. doi: 10.1105/tpc.108. 05827

Kühn, C., and Grof, C. P. (2010) Sucrose transporters of higher plants. Curr. Opin. Plant Biol. 13, 288-298. doi: 10.1016/j.pbi.2010. 02.001

Kühn, C., Quick, W. P., Schulz, A., Riesmeier, J. R., Sonnewald, U., and Frommer, W. B. (1996). Companion cell-specific inhibition of the potato sucrose transporter SUT1. Plant Cell Environ. 19, 1151123. doi: 10.1111/j.1365-3040.1996. tb00426.x

Kwik, J., Boyle, S., Fooksman, D., Margolis, L., Sheetz, M. P., and Edidin, M. (2003). Membrane cholesterol, lateral mobility, and the phosphatidylinositol 4,5-bisphosphate-dependent organization of cell actin. Proc. Natl. Acad. Sci. U.S.A. 100 13964-13969. doi: 10.1073/pnas. 2336102100
Lalonde, S., Sero, A., Pratelli, R., Pilot, G., Chen, J., Sardi, M. I., etal. (2010). A membrane protein/signaling protein interaction network for Arabidopsis version AMPv2. Front. Physiol. 1:24. doi: 10.3389/fphys.2010.00024

Leggewie, G., Kolbe, A., Lemoine, R., Roessner, U., Lytovchenko, A., Zuther, E., et al. (2003). Overexpression of the sucrose transporter SoSUT1 in potato results in alterations in leaf carbon partitioning and in tuber metabolism but has little impact on tuber morphology. Planta 217, 158-167.

Li, Y., Li, L. L., Fan, R. C., Peng, C. C., Sun, H. L., Zhu, S. Y., et al. (2012). Arabidopsis sucrose transporter SUT4 interacts with cytochrome b5-2 to regulate seed germination in response to sucrose and glucose. $\mathrm{Mol}$. Plant 5, 1029-1041. doi: 10.1093/ $\mathrm{mp} / \mathrm{sss} 001$

Liesche, J., He, H. X., Grimm, B., Schulz, A., and Kühn, C. (2010). Recycling of Solanum sucrose transporters expressed in yeast, tobacco, and in mature phloem sieve elements. Mol. Plant 3, 1064-1074. doi: $10.1093 / \mathrm{mp} / \mathrm{ssq} 059$

Loh, E., and Hong, W. (2002). Sec34 is implicated in traffic from the endoplasmic reticulum to the Golgi and exists in a complex with GTC-90 and ldlBp. J. Biol. Chem. 277, 2195521961. doi: 10.1074/jbc.M202326200 Lorente-Rodriguez, A., Heidtman, M. and Barlowe, C. (2009). Multicopy suppressor analysis of thermosensitive YIP1 alleles implicates GOT1 in transport from the ER. J. Cell Sci. 122, 1540-1550. doi: $10.1242 /$ jcs. 042457

Martiniere, A., Lavagi, I., Nageswaran, G., Rolfe, D. J., Maneta-Peyret, L., Luu, D. T., et al. (2012). Cell wall constrains lateral diffusion of plant plasma-membrane proteins. Proc. Natl. Acad. Sci. U.S.A. 109, 12805-12810. doi: 10.1073/pnas. 1202040109

Meyer, S., Lauterbach, C., Niedermeier, M., Barth, I., Sjolund, R. D., and Sauer, N. (2004). Wounding enhances expression of AtSUC3, a sucrose transporter from Arabidopsis sieve elements and sink tissues. Plant Physiol. 134, 684-693. doi: 10.1104/pp.103.033399

Mongrand, S., Stanislas, T., Bayer E. M., Lherminier, J., and SimonPlas, F. (2010). Membrane rafts in plant cells. Trends Plant Sci. 15, 656-663. doi: 10.1016/j.tplants.2010. 09.003

Nahirnak, V., Almasia, N. I., Fernandez, P. V., Hopp, H. E., Estevez, J.
M., Carrari, F., et al. (2012a). Potato snakin-1 gene silencing affects cell division, primary metabolism, and cell wall composition. Plant Physiol. 158, 252-263. doi: 10.1104/pp.111. 186544

Nahirnak, V., Almasia, N. I., Hopp, H. E., and Vazquez-Rovere, C. (2012b) Snakin/GASA proteins: involvement in hormone crosstalk and redox homeostasis. Plant Signal. Behav. 7, 1004-1008. doi: 10.4161/psb.20813

Niittyla, T., Fuglsang, A. T., Palmgren, M. G., Frommer, W. B., and Schulze, W. X. (2007). Temporal analysis of sucrose-induced phosphorylation changes in plasma membrane proteins of Arabidopsis. Mo Cell. Proteomics 6, 1711-1726. doi 10.1074/mcp.M700164-MCP200

Nühse, T. S., Stensballe, A., Jensen, O. N., and Peck, S. C. (2004). Phosphoproteomics of the Arabidopsis plasma membrane and a new phosphorylation site database. Plant Cell 16, 2394-2405. doi $10.1105 /$ tpc. 104.023150

Pilon, M., Schekman, R., and Romisch, K. (1997). Sec61p mediates export of a misfolded secretory protein from the endoplasmic reticulum to the cytosol for degradation. EMBO J. 16, 4540-4548. doi: 10.1093/emboj/16.15.4540

Rao Malla, R., Raghu, H., and Rao, J. S. (2010). Regulation of NADPH oxidase (Nox2) by lipid rafts in breast carcinoma cells. Int. J. Oncol. 37, 1483-1493.

Rapoport, T. A. (1992). Transport of proteins across the endoplasmic reticulum membrane. Science 258, 931-936.doi: 10.1126/science. 1332192

Reinders, A., Schulze, W., Kuhn, C. Barker, L., Schulz, A., Ward, J. M., et al. (2002a). Protein-protein interactions between sucrose transporters of different affinities colocalized in the same enucleate sieve element. Plant Cell 14, 1567-1577. doi: $10.1105 /$ tpc.002428

Reinders, A., Schulze, W., Thaminy, S., Stagljar, I., Frommer, W. B., and Ward, J. M. (2002b). Intra- and intermolecular interactions in sucrose transporters at the plasma membrane detected by the split-ubiquitin system and functional assays. Structure 10, 763-772. doi: 10.1016/S09692126(02)00773-6

Reuff, M., Mikosch, M., and Homann, U. (2010). Trafficking, lateral mobility and segregation of the plant K channel KAT1. Plant Biol. (Stuttg.) 12(Suppl. 1), 99104. doi: $10.1111 / \mathrm{j} .1438-8677.2010$. 00355.x
Roblin, G., Sakr, S., Bonmort, J., and Delrot, S. (1998). Regulation of a plant plasma membrane sucrose transporter by phosphorylation. FEBS Lett. 424, 165168. doi: 10.1016/S0014-5793(98) 00165-3

Roche, Y., Gerbeau-Pissot, P., Buhot, B., Thomas, D., Bonneau, L., Gresti, J., etal. (2008). Depletion of phytosterols from the plant plasma membrane provides evidence for disruption of lipid rafts. FASEB J. 22, 3980-3991. doi: 10.1096/fj.08111070

Rubinovich, L., and Weiss, D. (2010). The Arabidopsis cysteine-rich protein GASA4 promotes GA responses and exhibits redox activity in bacteria and in planta. Plant J. 64, 10181027. doi: 10.1111/j.1365-313X.2010. 04390.x

Schmitz, A., Herrgen, H., Winkeler, A., and Herzog, V. (2000). Cholera toxin is exported from microsomes by the Sec61p complex. J. Cell Biol. 148, 1203-1212. doi: 10.1083/jcb.148.6.1203

Schulz, A., Kühn, C., Riesmeier, J. R., and Frommer, W. B. (1998). Ultrastructural effects in potato leaves due to antisense-inhibition of the sucrose transporter indicate an apoplasmic mode of phloem loading. Planta 206, 533-543.

Schulze, W. X., Reinders, A., Ward, J., Lalonde, S., and Frommer, W. B. (2003). Interactions between co-expressed Arabidopsis sucrose transporters in the split-ubiquitin system. BMC Biochem. 4:3. doi: 10.1186/1471-2091-4-3

Schwachtje, J., Minchin, P. E., Jahnke, S., van Dongen, J. T., Schittko, U., and Baldwin, I. T. (2006). SNF1related kinases allow plants to tolerate herbivory by allocating carbon to roots. Proc. Natl. Acad. Sci. U.S.A. 103, 12935-12940. doi: 10.1073/pnas.0602316103

Shin, R., Jez, J. M., Basra, A., Zhang, B., and Schachtman, D. P. (2011). 14-3-3 proteins fine-tune plant nutrient metabolism. FEBS Lett. 585, 143-147. doi: 10.1016/ j.febslet.2010.11.025

Stadler, R., and Sauer, N. (1996). The Arabidopsis thaliana AtSUC2 gene is specifically expressed in companion cells. Bot. Acta 109, 299-306.

Stagljar, I., Korostensky, C., Johnsson, N., and te Heesen, S. (1998) A genetic system based on splitubiquitin for the analysis of interactions between membrane proteins in vivo. Proc. Natl. Acad. Sci. U.S.A. 95, 5187-5192. doi: 10.1073/pnas.95. 9.5187 
Vilhardt, F., and van Deurs, B. (2004). The phagocyte NADPH oxidase depends on cholesterol-enriched membrane microdomains for assembly. EMBO J. 23, 739-748. doi: 10.1038/sj.emboj.7600066

Wang, L., Wang, Z., Xu, Y., Joo, S. H., Kim, S. K., Xue, Z., et al. (2009). OsGSR1 is involved in crosstalk between gibberellins and brassinosteroids in rice. Plant J. 57, 498-510. doi: 10.1111/j.1365313X.2008.03707.x

Weise, A., Barker, L., Kühn, C., Lalonde, S., Buschmann, H., Frommer, W. B., et al. (2000). A new subfamily of sucrose transporters, SUT4, with low affinity/high capacity localized in enucleate sieve elements of plants. Plant Cell 12, 1345-1355.

Wigoda, N., Ben-Nissan, G., Granot, D., Schwartz, A., and Weiss, D. (2006). The gibberellin-induced, cysteine-rich protein GIP2 from Petunia hybrida exhibits in planta antioxidant activity. Plant J. 48 796-805. doi: 10.1111/j.1365-313X 2006.02917.x

Yang, X., Jiang, R., and Carlson, M. (1994). A family of proteins containing a conserved domain that mediates interaction with the yeast SNF1 protein kinase complex. EMBO J. 13, 5878-5886.

Conflict of Interest Statement: The authors declare that the research was conducted in the absence of any commercial or financial relationships that could be construed as a potential conflict of interest.

Received: 04 April 2013; accepted: 16 June 2013; published online: 02 July 2013.

Citation: Krügel U and Kühn C (2013)

Post-translational regulation of sucrose transporters by direct protein-protein interactions. Front. Plant Sci. 4:237. doi: 10.3389/fpls.2013.00237

This article was submitted to Frontiers in Plant Physiology, a specialty of Frontiers in Plant Science.

Copyright (c) 2013 Krügel and Kühn. This is an open-access article distributed under the terms of the Creative Commons Attribution License, which permits use, distribution and reproduction in other forums, provided the original authors and source are credited and subject to any copyright notices concerning any third-party graphics etc. 